\title{
EIderly Fall Detection Devices Using Multiple AIoT Biomedical Sensors
}

\author{
Cheng-Wen Lee ${ }^{1}$ and Hsiu-Mang Chuang ${ }^{2}$
}

\begin{abstract}
Due to the influence of degeneration and chronic diseases of elderly people, a higher chance of fall-related injuries occurs among them. Falling is one of the accidents frequently confronted by elderly people, so this issue is worthy of concern. We propose diverse models to analyze falls through a wearable device. Then, we use Artificial Intelligence of Things (AIoT) biomedical sensors for fall detection to build a system for monitoring elderly people's falls caused by dementia. The system can meet the safety needs of elderly people by providing communication, position tracking, fall detection, and pre-warning services. This device can be worn on the waist of an elderly people. Moreover, the device can monitor whether or not the person is walking normally, transmit the information to the rear-end system, and inform his/her family member via a cellphone app while an accident is occurring. Considering the risks on the fall test of elderly people, this study adopts activities of daily living (ADL) to verify the test. According to the test results, the accuracy of fall detection is $93.7 \%$, the false positive rate is $6.2 \%$, and the false negative rate is $6.5 \%$. To improve the accuracy of fall detection and the timely handling of appropriate referrals, may be highly expected to reduce the occurrence of fallrelated injuries.
\end{abstract}

JEL classification numbers: D61, I30, O32.

Keywords: Fall Detection, AIoT Sensor, Elderly People.

${ }^{1}$ College of Business, Chung Yuan Christian University, Taoyuan 320314, Taiwan.

${ }^{2}$ College of Business, Chung Yuan Christian University, Taoyuan 320314, Taiwan.

Article Info: Received: August 16, 2021. Revised: August 30, 2021.

Published online: September 3, 2021. 


\section{Introduction}

According to the report of Health Promotion Administration of Department of Health of Executive Yuan, Taiwan (2020), the ratio of people over 65 years old is $16.29 \%$ of the total population. In the past year, $20.5 \%$ of elderly people above the age of 65 years old suffered a fall, among which 37\% fell repeatedly. The data indicate that Taiwan has already entered an aging society (National Development Council, 2020). Within the aging of society, there is an increasing number of people with dementia, who are prone to falling (Albert et al., 2012). The aim of our research is to build fall detection and monitoring devices for elderly people and dementia patients, and then provide integrated innovative services for fall status assessment and personnel positioning through action-oriented devices, thus reducing the risk of accidents by the wearer.

Therefore, the main direction of our research is to distinguish fall events and the activities of daily living (ADL). Due to the high similarities of ADL such as sitting, standing, lying down, holding a wall, and falling slowly (Casilari, Santoyo-Ramn, and Cano-Garca, 2017), it is necessary to collect ADL data of the user and data generated by a volunteer simulating these actions, such as gravitational acceleration, angular velocity to analyze data, classify data, and improve accuracy. All fall analyses and detections can be covered by an actionable device.

In this study, by focusing on the fall detection of elderly people based on multiple AIoT sensors, we define six fall scenarios: fall forward, fall leftward, fall rightward, left back fall, right back fall, and tripping. An acceleration sensor and a gyroscope are used to check the action of the tester when receiving the warning from an Artificial Intelligence of Things (AIoT) sensor (Kangas et al., 2008).

Currently, the research and development of bracelets, intelligent garments, foot sensors, and other devices are ongoing. Although a wrist fall detector is easy to use, the wrist is not stable, so it is very difficult to analyze the shifting of weight with such a wrist fall detector. By contrast, the system of this study under current study is located near the gravity center of the human body (Lai et al., 2010), making the data highly stable. The ADL verification of this study will be explained in the following chapters.

\section{System Composition and Methods}

\subsection{System composition}

Following Igual, Medrano, and Plaza (2013), this study simulates the scenario in which an elderly people may fall (see Figure 1). We also use the testing device shown in Figure 2(a), which was worn in the position shown in Figure 2(b) based on Noury et al. (2007).

This AIoT device is composed of four main components:

1) An inertia measuring unit (IMU) containing an accelerometer (Gibson et al., 2015), and a gyroscope used for fall detection (Alarifi and Alwadain, 2021).

2) Global positioning system (GPS) for personal positioning.

3) Communication module for transmitting telecommunication and network 
information; according to the current plan, the device MTK MT2503A is used as the communication module of AIoT device.

4) Microcontroller unit (MCU) works like the CPU of personal computer and serves as the central control core of the whole AIoT device.

We use Sensortec BMI160 IMU (Bosch Sensortec Community, 2020), as the fall information search module of AIoT device (see Table 1).

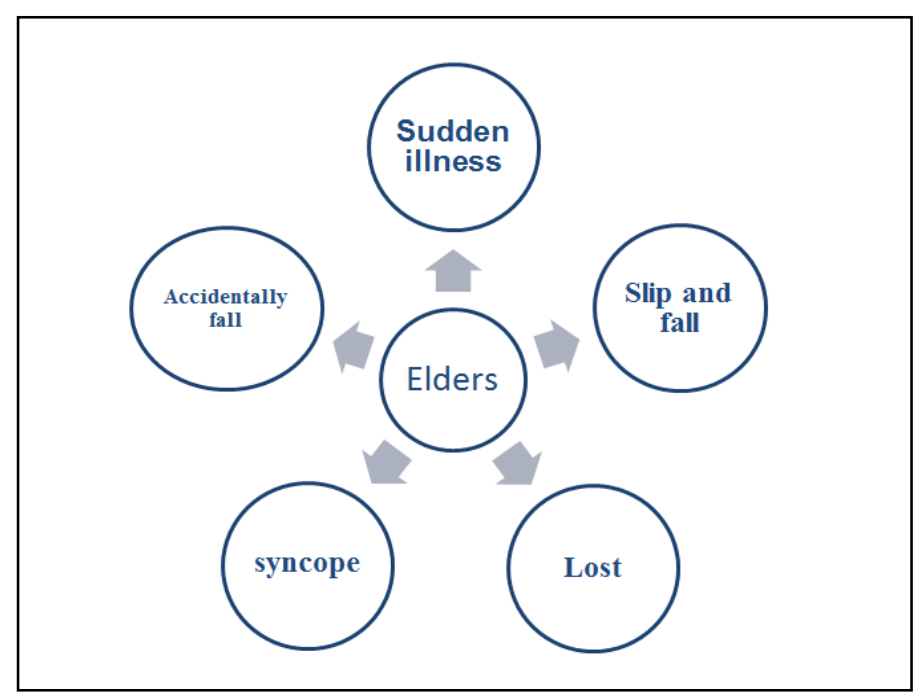

Figure 1: Risk factors for falls induced in elderly people

Source: Igual, Medrano, and Plaza (2013)

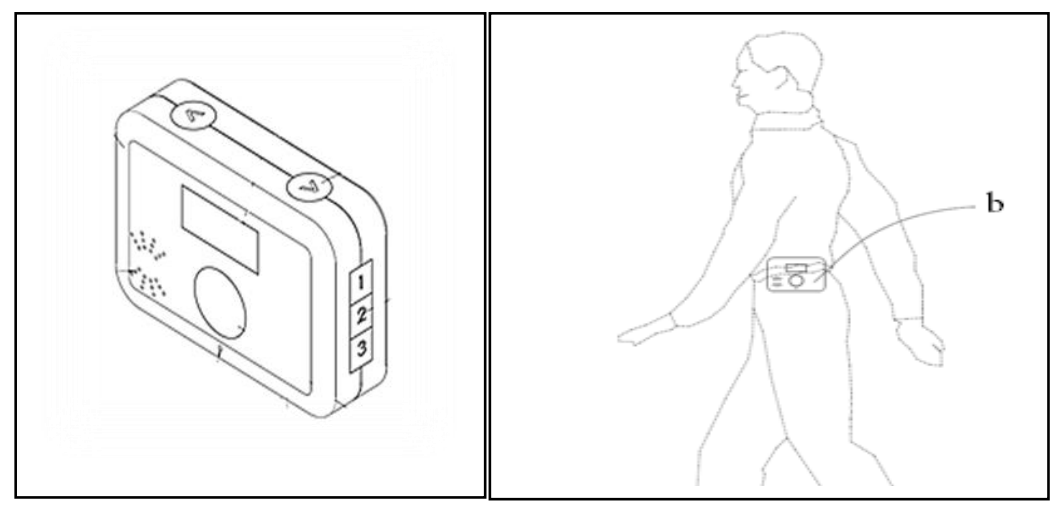

(a)

(b)

Figure 2: (a) Wearable device. (b) Position where device is worn

Source: Noury et al. (2007) 
Table 1: BMI160 IMU specifications

\begin{tabular}{|c|c|c|c|}
\hline Parameter & BMl160 & BMM150 & BMG250 \\
\hline Digital resolution & $\begin{array}{l}\text { Accelerometer (A): } 16 \text { bit } \\
\text { Gyroscope (G): } 16 \text { bit }\end{array}$ & $\begin{array}{c}\mathrm{X}, \mathrm{Y} \text { axis } 13 \text { bit } \\
\mathrm{Z} \text { axis } 15 \text { bit } \\
\mathrm{X}, \mathrm{Y}, \mathrm{Z} 32 \text { bit after API }\end{array}$ & Gyroscope (G): 16 bit \\
\hline Range and Sensitivity & $\begin{array}{c}\text { (A): } \pm 2 \mathrm{~g}: 16384 \mathrm{LSB} / \mathrm{g} \\
\quad \pm 4 \mathrm{~g}: 8192 \mathrm{LSB} / \mathrm{g} \\
\quad \pm 8 \mathrm{~g}: 4096 \mathrm{LSB} / \mathrm{g} \\
\quad \pm 16 \mathrm{~g}: 2048 \mathrm{LSB} / \mathrm{g} \\
(\mathrm{G}): \pm 125 \% \mathrm{~s}: 262.4 \mathrm{LSB} / \% \mathrm{~s} \\
\pm 250 \% \mathrm{~s}: 131.2 \mathrm{LSB} / \% \mathrm{~s} \\
\pm 500 \% \mathrm{~s}: 65.6 \mathrm{LSB} / \% \\
\pm 1000 \% \mathrm{~s}: 32.8 \mathrm{LSB} / \% \mathrm{~s} \\
\pm 2000 \% \mathrm{~s}: 16.4 \mathrm{LSB} / \% \mathrm{~s}\end{array}$ & $\begin{array}{l}X, Y: \pm 1300 \text { uT: } 0.0625 \text { uT } \\
Z: \pm 2500 \text { uT: } 0.0625 \text { uT }\end{array}$ & 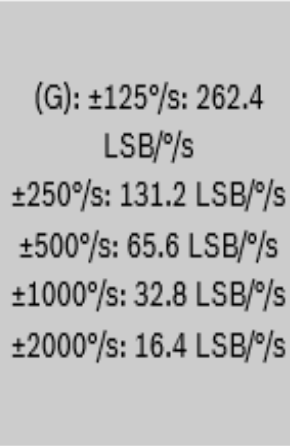 \\
\hline $\begin{array}{c}\text { Zero-g/rate/B offset (typ., } \\
\text { over life-time) }\end{array}$ & (A): $\pm 40 \mathrm{mg}(\mathrm{G}): \pm 10^{\circ} / \mathrm{s}$ & $\begin{array}{c}\text { RaW: } \pm 40 \text { uT } \\
\text { After calibration: } \pm 2 \text { uT }\end{array}$ & $(\mathrm{G}): \pm 10 \% \mathrm{~s}$ \\
\hline Noise density (typ.) & $\begin{array}{l}\text { (A): } 180 \mu \mathrm{g} / \sqrt{\mathrm{Hz}} \\
(\mathrm{G}): 0.008 \% \mathrm{~s} / \sqrt{\mathrm{H} z}\end{array}$ & NA & (G): $0.008 \% \mathrm{~s} / \sqrt{\mathrm{H} z}$ \\
\hline Bandwidths (programmable) & $1600 \mathrm{~Hz} \ldots 25 / 32 \mathrm{~Hz}$ & $300 \mathrm{~Hz} \ldots 5 \mathrm{~Hz}$ & $3200 \mathrm{~Hz} \ldots 25 \mathrm{~Hz}$ \\
\hline Interface & \multicolumn{3}{|c|}{$\left.\mathrm{SP} \&\right|^{2} \mathrm{C}, 2 \mathrm{x}$ digital interrupt pins } \\
\hline \multirow{2}{*}{ Supply voltage } & VDD: $1.71 \ldots 3.6 \mathrm{~V}$ & VDD: $1.62 \ldots 3.6 \mathrm{~V}$ & \\
\hline & & VDDIO:1.2 ... $3.6 \mathrm{~V}$ & \\
\hline Package & $2.5 \times 3.0 \times 0.8 \mathrm{~mm}^{3}$ & $1.56 \times 1.56 \times 0.6 \mathrm{~mm}^{3}$ & $2.5 \times 3.0 \times 0.8 \mathrm{~mm}^{3}$ \\
\hline Feature/Interrupt & $\begin{array}{c}\text { Step counter/step } \\
\text { detector/significant motion } \\
\text { Tap/double-tap } \\
\text { Any motion/no motion } \\
\text { Orientation/flat detection } \\
\text { Low-g/high-g detection }\end{array}$ & \multicolumn{2}{|c|}{ NA } \\
\hline
\end{tabular}

Source: Bosch Sensortec Community (2020)

Since the BMI160 IMU contains a telecom chip and a Bluetooth transmitter, it can also be regarded as an information gateway. Such a gateway can be connected to other Bluetooth devices to collect more physiological information of the wearer (such as heartbeat, temperature, blood oxygen, etc.) (Figure 3), and transmit it to the back-end system to provide medical staff with more physiological information on the wearer. 


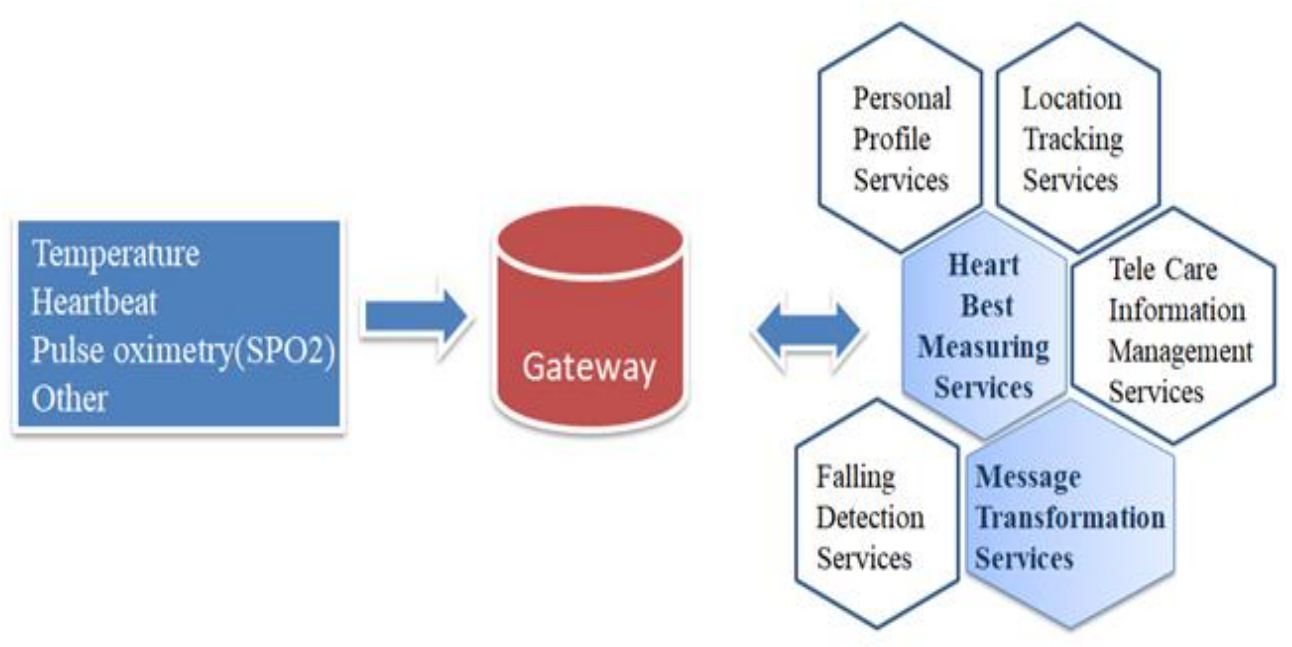

Figure 3: Physiological information gateway

\subsection{Evaluation methods}

The systematic fall detection mechanism designed by the study is detected by a dynamic sensor (such as accelerometer or gyroscope); the accelerometer measures the acceleration due to the ones that the accelerometer measures the accelerated velocity along three axles $(x, y$, and $z)$ and the gyroscope measures the instantaneous angular velocity around three axes $(x, y$, and $z)$. The change in body posture (such as a change in the body's fall speed, status and direction) is used for determining whether a person falls or not. The most well-known fall analysis algorithm is the one proposed by Mathie et al. (2002), they calculated the signal intensity vector/signal vector magnitude (SVM) and configured the combined acceleration strength vector; the SVM is given by Equation (1). Later, an improvement was made and an analysis was made via SMV referring to the Equation (2), where $A_{x}(t), A_{y}(t)$ and $A_{z}(t)$ are respectively the acceleration magnitude of the time $\mathrm{t}$ on the axis $x, y$, and $z$. The SMV exceeded the threshold (acquiring the upper limit and the lower limit), plenty of ADL test data showed the fall. , In terms of calculation, we mainly use the characteristic value SMV as the characteristic quantity to reflect the acceleration of the human body. Acceleration is vector and directional, so SMV can ignore the influence of the directions of acceleration, which is good for judging the same gesture in different directions. This method is still being widely used nowadays.

$$
\begin{aligned}
& S V M=\sqrt{a_{x}^{2}+a_{y}^{2}+a_{z}^{2}} \\
& S M V(t)=\sqrt{A_{x}(t)^{2}+A_{y}(t)^{2}+A_{z}(t)^{2}}
\end{aligned}
$$


We adopt the AIoT device that was made especially as the information carrier to link various Bluetooth actionable devices capable of searching for and collecting body signs. The hardware architecture diagram of this AIoT device is shown in Figure 4.

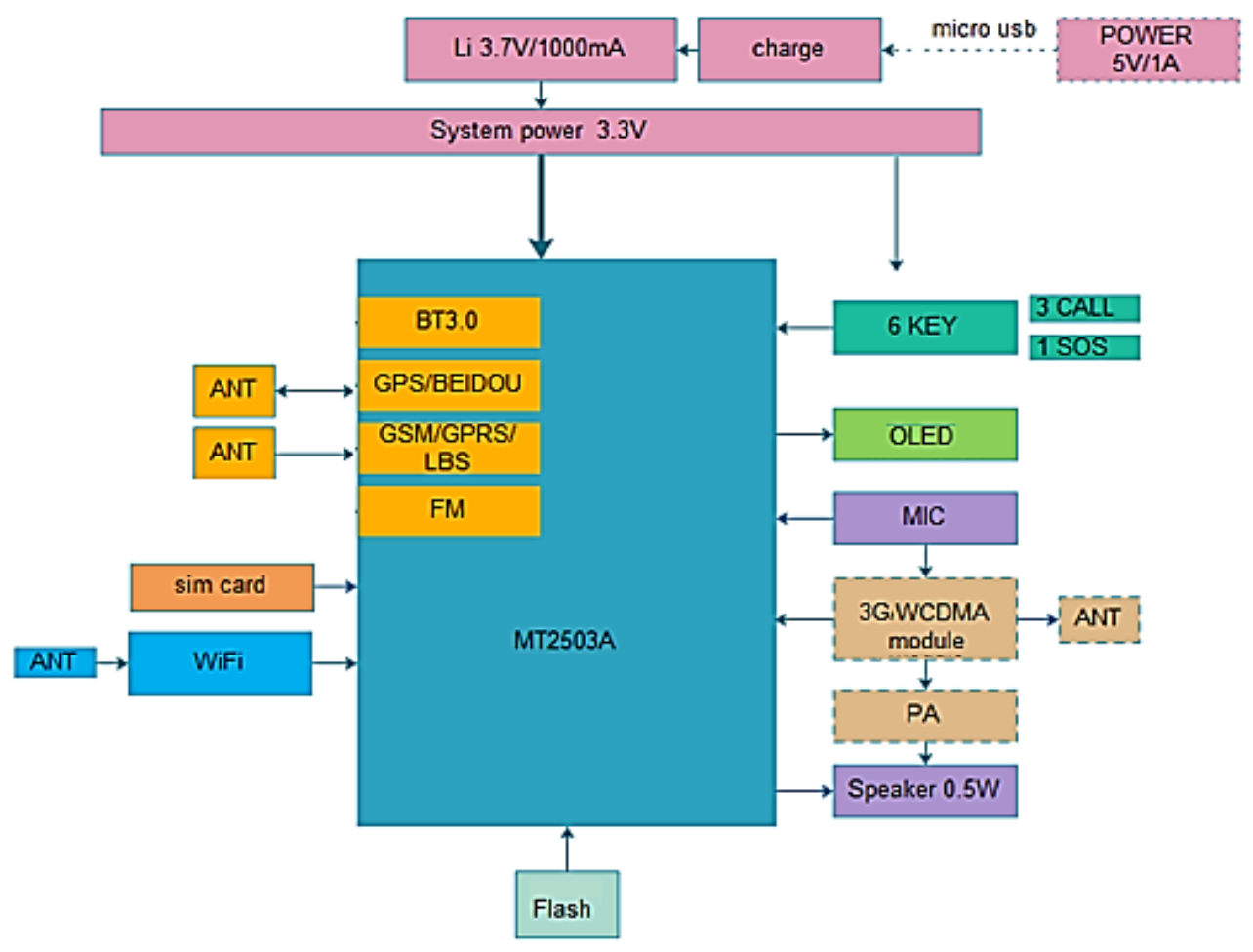

Figure 4: AIoT device architecture diagram

The following is a description of the main functions of this device:

1) Telecommunication communication, call function, capable of communication by telephone in an emergency.

2) GPS positioning and WiFi connection function, usable for automatic changeover of telecommunication communication and data transmission by WiFi indoors and outdoors.

3) Built-in acceleration gauge and gyroscope, capable of providing related parameters for evaluating fall status.

4) Built-in Bluetooth receiver for connecting Bluetooth device, capable of receiving payload data transmitted by other Bluetooth devices, and uploading to the cloud service by the telecommunication transmission port of carrier (this is the significance of the "action information carrier"). In this study, the carrier focuses on transmitting the original position and fall data to the cloud service. 
The MT2503A chip is designed with GPS, WiFi, Bluetooth, and a telecommunication transmission port; thus, the hardware planning of a wearable device should focus on the design of the firmware, device module, and structure. In this paper we mainly discuss fall detection based on multiple AIoT biomedicine sensors.

The action device can be detected by a dynamic sensor such as an accelerometer or gyroscope (Alarifi and Alwadain, 2021). An accelerometer focuses on measuring the acceleration along three axes $(x, y$, and $z$ ) including the result of gravitational acceleration (Zhang and Zhu, 2018), the gyroscope focuses on measuring the instantaneous angular velocity of rotation around the axes $(x, y$, and $z)$. The change in body posture (such as a change in the body's velocity and direction) is used for determining whether a person falls or not (Nooruddin, Islam, and Sharna, 2020). Currently, for the fall analysis algorithm, we refer to the studies (Du, Yin, and Zhang, 2015). The SMV method can measure the value of different body actions to some extent (See Table 2). In Ref. 13, the calculated SVM was proposed as a measure of the fall strength. When SVM exceeds a threshold (an upper limit or lower limit generated by volunteer testing), it can be judged that a fall occurs (Bagala et al., 2012).

Table 2: SMV values of ADL

\begin{tabular}{|c|c|c|c|c|}
\hline Standing & Walking & Brisk Walking & Sitting down & Falling down \\
\hline $1.03 \mathrm{~g}$ & $1.17-1.25 \mathrm{~g}$ & $1.27-1.45 \mathrm{~g}$ & $1.5-1.6 \mathrm{~g}$ & $1.87-2.4 \mathrm{~g}$ \\
\hline
\end{tabular}

The SVM algorithm judges whether a fall occurs; when the data exceeds a specific threshold, it is judged that a fall occurs, so a warning will be triggered. This approach has the following problems:

1) The advantage of using an accelerometer is to measure the component of gravitational acceleration along three axes and enter the mode with lowest electric power consumption under the condition that there is no motion or vibration (idle state). However, its disadvantages are that not all attitude angles can be deduced and the dynamic response is rather slow.

2) The specific thresholds are usually decided by performing simulated tests on young volunteers, but the actual threshold of elderly people or patients may not be the same.

Referring to the Equation (2), we adopted tilt angle (TA) as the analysis parameter to improve the reading accuracy referring to the Equation (3).

$$
\mathrm{TA}=\sin ^{-1}\left(\frac{a_{\mathrm{y}}}{\sqrt{a_{\mathrm{x}}^{2}+a_{\mathrm{y}}^{2}+a_{\mathrm{z}}^{2}}}\right)
$$




\subsection{Falling status analysis and evaluation}

After analyzing the falling types (for instance: fall forward, fall backward, fall sideward, tumble, fall slowly by sustaining wall, and slipping etc.), this study divides the fall process into four states (weightlessness, impact, stationary, and comparison with the initial state) (as illustrated in Figure 5).

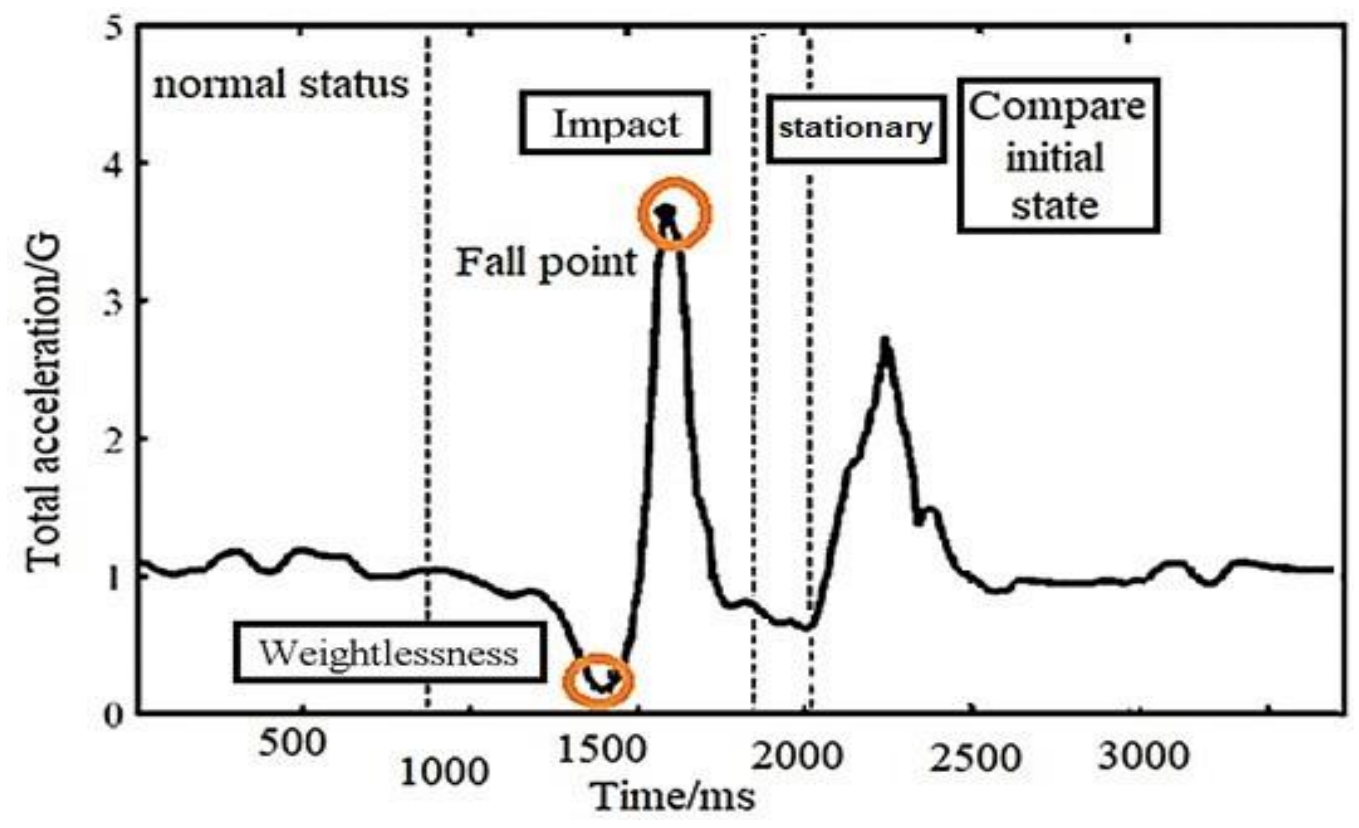

Figure 5: The change in the amplitude of the accelerometer data during a fall

The case as shown in Figure 5 is the one that a person who can pick him up and thereby continue his following action after he falls down; if a person falls down and remains unconscious, or can not return to normal after that, the system will trigger an alarm in a given time (generally 15 seconds). However, according to related studies (Stone and Skubic, 2015), when an elderly people falls down, he may keep his staying on the ground for gasp, whereas the system will judge the information generated by the relevant behavior and which will display the importance. In our study, we divide the general fall process into 4 stages.

\section{Weightlessness}

At the beginning of a fall, the body will fall down or slide. When the acceleration magnitude changes suddenly, the tilting angle of the body may rapidly increase. Referring to the Table 2, the measured data of acceleration gauge during the fall period ranges from $1.87 \mathrm{~g}$ to $2.4 \mathrm{~g}$, but such range, whether it fits to the scenarios of costive elderly people, namely fall in a faint and fall down at the bedside (in a dangerous situation), walk along the staircase from top to bottom (not in a dangerous situation), judge as the fall sideward (in a dangerous situation) or do morning exercises (not in a dangerous situation), may be corrected according to the case that may actually happen in future. 


\section{Impact}

When falling down, the body of a person will hit against the ground or another object, reversing the direction in the acceleration curve. This can be used as the second basis for the judging the state of falling.

\section{Stationary}

According to the practical experience of carer, an elderly people may stop his action and hold his temporary gesture when lying flat, such as support with hand or other auxiliary, or support by sustaining wall, or kneel down etc., such scenarios is concluded as the stationary state in the current study.

\section{Comparison with the initial state}

In the stationary state, if the information received by sensor fluctuates hugely, or returns to its initial status, we can presume that the wearer does not suffer with heavier injury; if the stationary state lasts for tens of seconds, or turns into the quiescent state, the system will trigger an alarm and will inform the relevant persons. The analysis herein focuses on the one reducing the occurrence rate of false alarm and reducing the burden of carer.

By synthesizing the four statuses aforesaid, the AIoT is designed with the fall interpretation algorithm in its MCU module, see Figure 6 for its process.
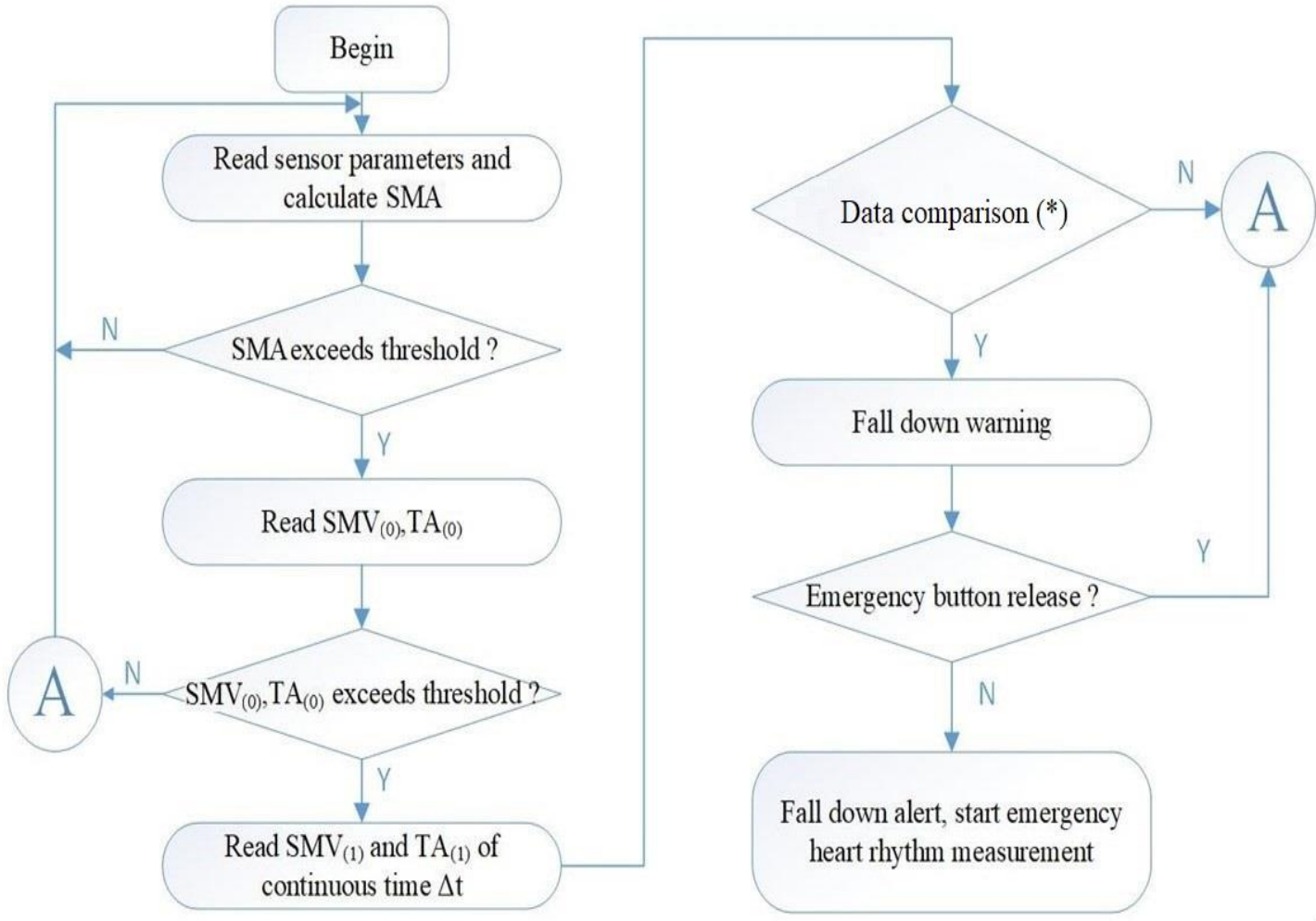

\section{Figure 6: Fall scenario analysis flowchart}

(*)By comparing the data collected in a given time, we can judge whether the features of four statuses are met, and whether $\operatorname{SMV} \Delta$ (i) and TA $\Delta$ (i) exceed their respective critical values in the condition of weight loss or impact. 


\section{Experiments}

At the beginning of working out the plan, we interviewed with the employees of elderly people's home and knew their troubles when frequently receiving false alarm. Sometimes the trouble is so severer than the one missing true alarm. In this plan, we aimed at obtaining the threshold value as required in the previous chapter by 4 young persons wearing AIoT device by the repeated and appointed action, and then verified the accuracy of alarm by repeating the same action of additional 5 young persons.

In the experimental process, testees were required to repeat the appointed action. However the data therefrom was different every time. In the process, false alarm may occur. In order to mark the false alarm and correct the threshold value of AIoT device, we designed an "Alarm off Key", it has the functions as follows:

(1)When triggering the fall alarm and after the wearer presses the "alarm off" key, the record will be kept in the application program, and such data will be marked as the "wrong alarm".

(2)When triggering the fall alarm, but the wearer does not press the "alarm off" key, the record will be kept in the application program, and such data will be marked as the "true alarm".

The falling status evaluation algorithm requires the test steps as follows (Lie, Le, and Lin, 2018; Meng et al., 2015; Rakhman et al., 2014).

\section{Part 1 Fall simulation}

1. Fall forward.

2. Fall leftward.

3. Fall rightward.

4. Left back fall.

5. Right back fall.

6. Tripping.

\section{Part 2 Common behavior}

7. Jump for $10 \mathrm{~min}$.

8. Brisk walking for $20 \mathrm{~min}$.

9. Daily wear for $2 \mathrm{~h}$.

The 7th item, i.e.: jump, refers to the slight jump when doing the morning exercises. We integrates the item by referring to other studies (Meng et al., 2015). The falling status evaluation algorithm requires following the test steps:

Tests 1-6: 100 ADL tests were conducted for each item.

Tests 7-9: 50 person-times for each item.

As for the relevant test verifications, see Table 3. 
Table 3: ADL verification

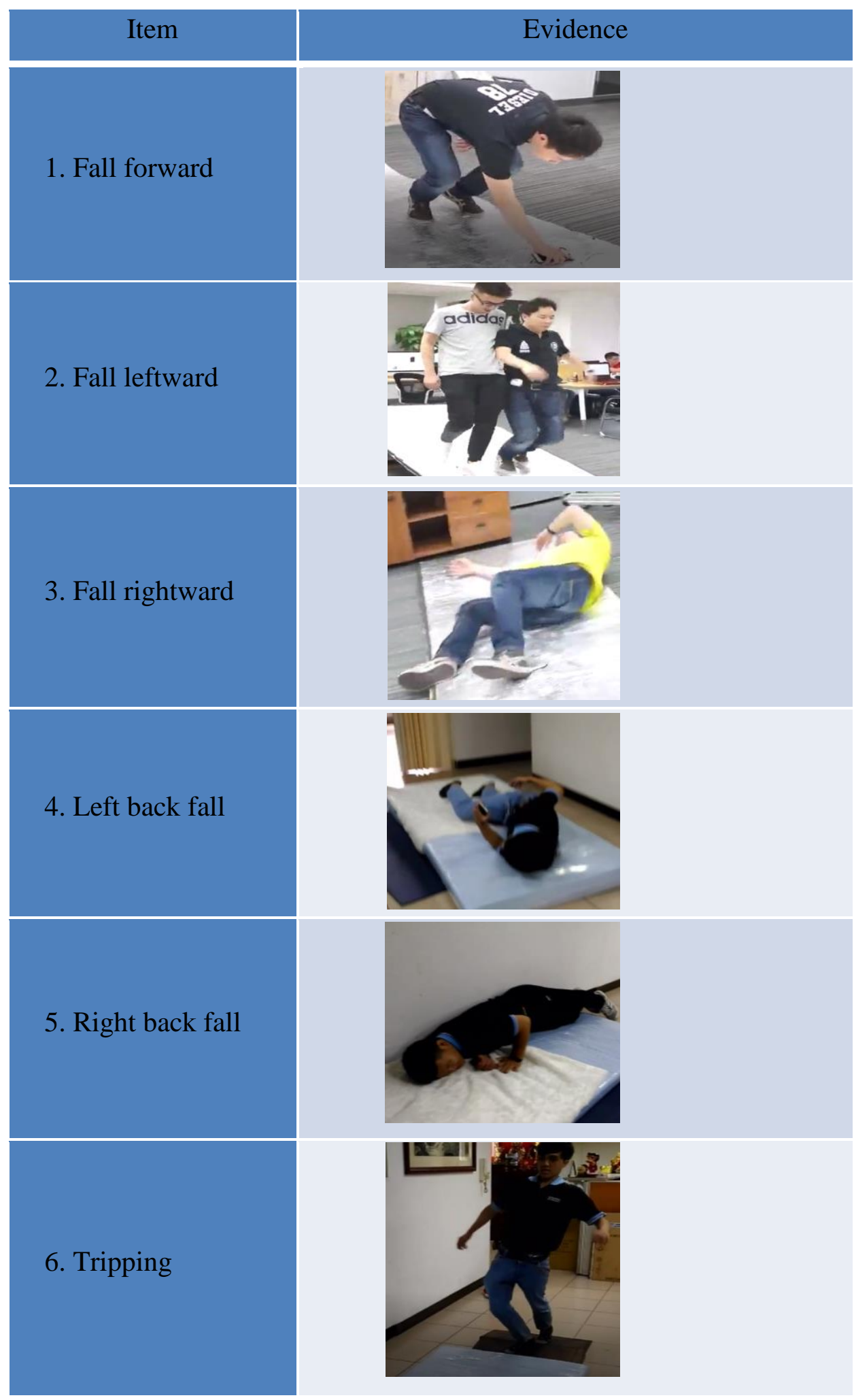


From the falling status evaluation algorithm requirements, we know the following: Tests 1-6: perform 100 ADL tests for every item if accuracy (ACC) $\geq 90 \%$, false positive rate $(\mathrm{FPR}) \leq 10 \%$, and false negative rate $(\mathrm{FNR}) \leq 10 \%$. The total number of ADL tests performed to evaluate the falling status is $1-6$, our actual number of samples is 660 person-times, and the effective sample number is 600 person-times. Table 4 shows the results.

True positive (TP): Number of falls detected in the fall sample (true alarm)

True negative (TN): Number of non-falling samples detected as not falling (no alarm)

False positive (FP): Number of falls detected in the non-falling samples (false alarm)

False negative (FN): Number of no falls detected in fall samples (no alarm)

- $\mathrm{ACC}($ Accuracy $)=(\mathrm{TP}+\mathrm{TN}) /(\mathrm{TP}+\mathrm{TN}+\mathrm{FP}+\mathrm{FN})$

- $\mathrm{FPR}=\mathrm{FP} /(\mathrm{FP}+\mathrm{TN})$

- $\mathrm{FNR}=\mathrm{FN} /(\mathrm{TP}+\mathrm{FN})$

Table 4: Experimental results

\begin{tabular}{|c|c|c|}
\hline ACC & $(\mathrm{TP}+\mathrm{TN}) /(\mathrm{TP}+\mathrm{TN}+\mathrm{FP}+\mathrm{FN})$ & $93.7 \%$ \\
\hline FPR & $\mathrm{FP} /(\mathrm{FP}+\mathrm{TN})$ & $6.2 \%$ \\
\hline FNR & $\mathrm{FN} /(\mathrm{TP}+\mathrm{FN})$ & $6.5 \%$ \\
\hline
\end{tabular}

\section{Field Domain Test}

In this study, we cooperated with long-term care organizations and used the help of elderly people's relatives and carers to make practical demand verification on the one that elderly people uses such system, and made adjustment by referring to other studies and comments of other relevant personnel in the process.

When the user wears AIoT device, if occurring fall accident in the normal condition, it may not immediately trigger an alarm toward the rear-end system, but may broadcast or call an emergency contact person (according to the default sequence) until it is answered. Thus, a family member can track the latest condition of the wearer by cellphone.

Employees of nursing homes can use the back-end part of the system to understand and track the condition of the wearer (Robinovitch et al., 2013). After installing a Bluetooth beacon in a nursing home, if the elderly people stay in the dangerous area defined by the nursing home for a long time, the back-end system may also alarm.

\section{Conclusions}

As the AIoT technology improves, an alarm service combined with wearable device's information and action information has already been aroused the attention of people in recent years. At present, in Taiwan, an aging society, the focus of medical services is shifting from emergency to chronic disease treatment and safety 
and health care. In this work, we propose AIoT device that is designed with a rearend system to facilitate communication, as well as a position tracking function, a fall detection function, and a pre-warning service, so the operator can be assured to master the information of monitoring site. For instance, in a family or an elderly people's nursing home, an alarm may be triggered by telephone whenever the wearer falls, so his/her family member or the on-duty operator can be informed and handle the emergency, thus reducing the number of people injured by falls.

The remote safety protection device used for monitoring and detecting falls allows the use of information and communication technology for accident prevention, health care, medical treatment, and other related services. It provides a remote health care service to assist in the health management of people with chronic illness, so it can make users produce self-control awareness and thereby improve the effectiveness of medical treatment and health care.

\section{References}

[1] Alarifi, A. and Alwadain, A. (2021). Killer heuristic optimized convolution neural network-based fall detection with wearable IoT sensor devices. Measurement, 167, 2021, p. 108258.

[2] Albert, M.V., Kording, K., Herrmann, M., and Jayaraman, A. (2012). Fall classification by machine learning using mobile phones. PLoS One, 7, p. e36556.

[3] Bagala, F., Becker, C., Cappello, A., Chiari, L., Aminian, M., Hausdorff, J.M., Zijlstra, W., and Klenk, J. (2012). Evaluation of accelerometer-based fall detection algorithms on real-world falls. PLoS ONE , 7(5), p. e37062.

[4] Bosch Sensortec Community (2020). BMI160 Series IMU Design Guide. https://community.bosch-sensortec.com/t5/Knowledge-base/BMI160-SeriesIMU -Design-Guide/ta-p/7376 (Accessed: Oct. 15, 2020).

[5] Casilari, E., Santoyo-Ramn, J. A., and Cano-Garca, J. M. (2017). UMAFall: A multisensor dataset for the research on automatic fall detection. Procedia Computer Science, 110, pp. 32-39.

[6] Du, B., Yin, L., and Zhang, Z. (2015). Application of support vector machines arithmetic in body posture detection. Proceedings of the 4th International Conference on Information Technology and Management Innovation, pp. 680684.

[7] Gibson, R. M., Amira, A., Ramzan, N., de-la Higuera, P. C., and Pervez, Z. (2016). Multiple comparator classifier framework for accelerometer-based fall detection and diagnostic. Applied Soft Computing, 39, pp. 94-103.

[8] Igual, R., Medrano, C., and Plaza, I. (2013). Challenges, issues and trends in fall detection systems. BioMedical Engineering OnLine, 12(6), pp.1-24.

[9] Kangas, M., Konttila, A., Lindgren, P., Winblad, I., and Jämsä, T. (2008). Comparison of low-complexity fall detection algorithms for body attached accelerometers. Gait \& Posture, 28(2), pp. 285-291. 
[10] Lai, C. F., Huang, Y. M., Park, J. H., and Chao, H. C. (2010). Adaptive body posture analysis for elderly-falling detection with multisensors. IEEE Intelligent Systems, 25(2), pp. 20-30.

[11] Lie, W. N., Le, A. T., and Lin, G. H. (2018). Human fall-down event detection based on 2D skeletons and deep learning approach. 2018 International Workshop on Advanced Image Technology (IWAIT), pp. 1-4.

[12] Mathie, M. J., Lovell, N. H., Coster, A. C. F., and Celler, B. G. (2002). Determining activity using a triaxial accelerometer. Proceedings of the Second Joint EMBS/BMES Conference, Houston, TX. USA October 23-26.

[13] Meng, W., Wang, C., Li, H. and Ji, M. (2015). Fall detection algorithm for the elderly based on trial acceleration and heart rate. Proceedings of the 2015 3rd International Conference on Machinery, Materials and Information Technology Applications, pp. 137-142.

[14] National Development Council (2020). Population projections for the R.O.C. https://pop-proj.ndc.gov.tw/chart.aspx?c=10\&uid=66\&pid=60 (Accessed: Oct. 17, 2020).

[15] Nooruddin, S., Islam, M. M., and Sharna, F. A. (2020). An IoT based devicetype invariant fall detection system. Internet of Things, 9 (100130), pp. 1-18. https://doi.org/10.1016/j.iot.2019.100130

[16] Noury, N., Fleury, A., Rumeau, P., Bourke, A. K., Laighin, G. O., Rialle, V., and Lundy, J. E. (2007). Fall detection: Principles and methods. 2007 29th Annual International Conference of the IEEE Engineering in Medicine and Biology Society, pp, 1663-1666.

[17] Rakhman, A. Z., Nugroho, L. E., Widyawan, W., and Kurnianingsih, A. (2014). Fall detection system using accelerometer and gyroscope based on smartphone. 2014 The 1st International Conference on Information Technology, Computer, and Electrical Engineering, pp. 99-104.

[18] Robinovitch, S., Feldman, F., Yang, Y., Sims-Gould, J. and Leung, M. (2013). Falls in older people in long-term care: Authors' reply. The Lancet, 381(9873), p. 1180.

[19] Stone, E. E. and Skubic, M. (2015). Fall detection in homes of older adults using the Microsoft Kinect. IEEE Journal of Biomedical and Health Informatics, 19(1), pp. 290-301.

[20] Zhang, Q. and Zhu, S. (2018). Real-time activity and fall risk detection for aging population using deep learning. 2018 9th IEEE Annual Ubiquitous Computing, Electronics \& Mobile Communication Conference (UEMCON), pp. 1055-1059. 